\title{
An Efficient Computational Method for Singularly Perturbed Delay Parabolic Partial Differential Equations
}

\author{
${ }^{1}$ Imiru Takele Daba, ${ }^{2}$ Gemechis File Duressa \\ ${ }^{1}$ Wollega University Department of Mathematics Nekemte, Oromia ETHIOPIA \\ ${ }^{2}$ Jimma University Department of Mathematics, Jimma, Oromia ETHIOPIA
}

Received: May 24, 2021. Revised: July 8, 2021. Accepted: July 12, 2021. Published: July 21, 2021.

\begin{abstract}
In this communication, a parameter uniform numerical scheme is proposed to solve singularly perturbed delay parabolic convection-diffusion equations. Taylor's series expansion is applied to approximate the shift term. Then the resulting singularly perturbed parabolic convection-diffusion equation is solved by utilizing the implicit Euler method for temporal discretization on uniform mesh and hybrid numerical scheme based on a midpoint upwind scheme in the coarse mesh regions and a cubic spline method in the fine mesh regions on a piecewise uniform Shishkin mesh for the spatial discretization. The proposed numerical scheme is shown to be an $\varepsilon$-uniformly convergent accuracy of first-order in time and almost second-order in space directions. Some test examples are considered to testify the theoretical predictions.
\end{abstract}

Key-Words: Singular perturbation problem; Delay parabolic differential equations; Implicit Euler method; Cubic spline difference method; Hybrid numerical scheme.

\section{INTRODUCTION}

In this paper, we consider the following model problem on the domain $D=\Omega_{x} \times \Omega_{t}=(0,1) \times(0, T]$ :

$$
\left\{\begin{array}{l}
\frac{\partial u}{\partial t}-\varepsilon^{2} \frac{\partial^{2} u}{\partial x^{2}}+\lambda(x) \frac{\partial u}{\partial x}+\nu(x) u(x-\delta, t) \\
+\varrho(x) u(x, t)=\eta(x, t),(x, t) \in D \\
u(x, 0)=u_{0}(x), x \in \bar{\Omega}_{x} \\
u(x, t)=\Upsilon_{1}(x, t),-\delta \leq x \leq 0, t \in \bar{\Omega}_{t} \\
u(x, t)=\Upsilon_{2}(x, t)
\end{array}\right.
$$

where $0<\varepsilon \ll 1$ is a singular perturbation parameter and $\delta$ is a delay parameter of $o(\varepsilon)$. The functions $\lambda(x), \nu(x), \varrho(x), \eta(x), \Upsilon_{1}(x), \Upsilon_{2}(x)$ and $u_{0}(x)$ are considered to be sufficiently smooth, bounded and independent of $\varepsilon$. We also considered $\nu(x)+\varrho(x) \geq$ $\zeta>0 \forall x \in \bar{\Omega}_{x}$, for some positive constant $\zeta$.

The considered above problem plays a prominent role in mathematical modeling of various practical phenomena such as in modeling of the neuronal variability [1], bistable devices [2], evolutionary biology [3], variational problems of control theory [4], to describe the human pupil-light reflex [5], and many more.

Due to the existence of $\varepsilon$ and delay in problem (1) make the problem tiresome to solve theoretically. Hence, to solve this problem, one has to look for sounding numerical methods. Solving problem (1) problem using the classical methods on a uniform mesh, unable to provide an efficient numerical solution until we use $h \ll \varepsilon$, where $h$ is the spatial step length. This drawback encourages researchers to develop the concept of $\varepsilon$-uniform numerical methods for such types of problems. In this context, the fitting techniques (operator and layer-adapted mesh ) are a competitive computational technique to overcome the limitations of classical methods.

Since the past decade, some scholars have been developing $\varepsilon$-uniform numerical methods for a class of time-dependent singularly perturbed delay parabolic partial differential equations (SPDPPDEs) with shift parameter(s) in the space variable and analyzing the effects of the shift parameters on the solution behavior. For instance, [6], [7], [8], [9], [10], [11], [12], [13] have developed different numerical methods based on fitting techniques for solving the SPDPPDEs and elucidated the effect of the shift arguments on the boundary layer behavior of the solution.

However, numerical methods to solve a class of time-dependent SPDPPDE with delay in the spatial variable having an $\varepsilon$-uniform convergence for the past decades are still few. The main contribution of this work is to construct an $\varepsilon$-uniform numerical scheme for solving the SPDPPDEs with delay parameter in the space variable. 


\section{A. Properties of the continuous solution}

When $\delta<\varepsilon$, the use of Taylor's series expansion for the terms containing shift arguments is valid [14]. Consequently, we considered this case, and applying Taylor's series expansion, we obtain

$$
u(x-\delta, t)=u(x, t)-\delta \frac{\partial u(x, t)}{\partial x}+O\left(\delta^{2}\right) .
$$

Now inserting Eq. (2) into Eq. (1), we obtain

$$
\left\{\begin{array}{l}
L_{\varepsilon} u(x, t)=\eta(x, t) \\
u(x, 0)=u_{0}(x), x \in \bar{\Omega}_{x} \\
u(0, t)=\Upsilon_{1}(0, t), t \in \bar{\Omega}_{t} \\
u(1, t)=\Upsilon_{2}(1, t), t \in \bar{\Omega}_{t}
\end{array}\right.
$$

where, $\quad L_{\varepsilon} u(x, t)=\frac{\partial u(x, t)}{\partial t}-\varepsilon^{2} \frac{\partial^{2} u(x, t)}{\partial x^{2}}+$ $\theta(x) \frac{\partial u(x, t)}{\partial x}+\vartheta(x) u(x, t), \theta(x)=\lambda(x)-\delta \nu(x)$ and $\vartheta(x)=\nu(x)+\varrho(x)$. Since $\theta(x) \geq \theta^{*}>0$ and $\vartheta(x) \geq \vartheta^{*}>0$ for some constants $\theta^{*}$ and $\vartheta^{*}$ the solution of Eq. (3) exhibits an exponential boundary layer at $x=1$. For small $\delta$, Eq. (1) and Eq. (3) have an almost equal approximate solution.

To elude conflict between boundary and initial condition, we assume the compatibility conditions on the corner of the domain $(0,0)$ and $(0,1)$ as [15]

$$
u_{0}(0)=\Upsilon_{1}(0,0), \quad u_{0}(1)=\Upsilon_{2}(1,0),
$$

and

$$
\left\{\begin{array}{l}
\frac{\partial \Upsilon_{1} i(0,0)}{\partial t}-\varepsilon^{2} \frac{\partial^{2} u_{0}(0)}{\partial x^{2}}+\theta(0) \frac{\partial u_{0}(0)}{\partial x}+\vartheta(0) u_{0}(0) \\
=\gamma(0,0) \\
\frac{\partial \Upsilon_{2}(1,0)}{\partial t}-\varepsilon^{2} \frac{\partial^{2} u_{0}(1)}{\partial x^{2}}+\theta(1) \frac{\partial u_{0}(1)}{\partial x}+\vartheta(1) u_{0}(1) \\
=\gamma(1,0)
\end{array}\right.
$$

Lemma 1 (Continuous Maximum Principle). Let $\Xi(x, t) \in C^{2,1}(\bar{D})$. If $\Xi(x, t) \geq 0, \forall(x, t) \in \partial D$ $(\partial D=\bar{D}-D)$ and $L_{\varepsilon} \Xi(x, t) \geq 0, \forall(x, t) \in D$, then $\Xi(x, t) \geq 0, \forall(x, t) \in \bar{D}$.

Proof: Let $\left(x^{*}, t^{*}\right) \in \bar{D}$ be such that

$$
\Xi\left(x^{*}, t^{*}\right)=\min _{(x, t) \in \bar{D}} \Xi(x, t)
$$

and suppose that $\Xi\left(x^{*}, t^{*}\right)<0$, then we have $\left(x^{*}, t^{*}\right) \notin \partial D$. Also we have $\frac{\partial \Xi\left(x^{*}, t^{*}\right)}{\partial x}=$ $0, \frac{\partial \Xi\left(x^{*}, t^{*}\right)}{\partial t}=0$, and $\frac{\partial^{2} \Xi\left(x^{*}, t^{*}\right)}{\partial x^{2}} \geq 0$. Then

$$
\begin{aligned}
& L_{\varepsilon} \Xi\left(x^{*}, t^{*}\right)=\frac{\partial \Xi\left(x^{*}, t^{*}\right)}{\partial t}-\varepsilon^{2} \frac{\partial^{2} \Xi\left(x^{*}, t^{*}\right)}{\partial x^{2}} \\
& +\theta\left(x^{*}\right) \frac{\partial \Xi\left(x^{*}, t^{*}\right)}{\partial x}+\vartheta\left(x^{*}\right) \Xi\left(x^{*}, t^{*}\right)<0
\end{aligned}
$$

which contradicts the assumption made above. It follows that $\Xi\left(x^{*}, t^{*}\right) \geq 0$ and hence $\Xi(x, t) \geq$ $0, \forall(x, t) \in \bar{D}$.
Lemma 2 (Stability estimate). The solution $u(x, t)$ of Eq. (3) satisfies

$$
\begin{aligned}
& \|u\| \leq\left(\vartheta^{*}\right)^{-1}\|\eta\| \\
& +\max \left\{\left|u_{0}(x)\right|, \max \left\{\left|\Upsilon_{1}(x, t)\right|,\left|\Upsilon_{2}(x, t)\right|\right\}\right\},
\end{aligned}
$$

where, $\|\cdot\|$ is the $L_{\infty}$ norm given by $\|u\|=$ $\max _{(x, t) \in \bar{D}}|u(x, t)|$.

Proof: Let $\Xi^{ \pm}(x, t)$ be two barrier functions defined by

$$
\begin{aligned}
& \Xi^{ \pm}(x, t)=\left(\vartheta^{*}\right)^{-1}\|\eta\| \\
& +\max \left\{\left|u_{0}(x)\right|, \max \left\{\left|\Upsilon_{1}(x, t)\right|,\left|\Upsilon_{2}(x, t)\right|\right\}\right\} \\
& \pm u(x, t) .
\end{aligned}
$$

Then at the initial value and the two end points, we have

$$
\begin{aligned}
& \Xi^{ \pm}(x, 0)=\left(\vartheta^{*}\right)^{-1}\|\eta\| \\
& \quad+\max \left\{\left|u_{0}(x)\right|, \max \left\{\left|\Upsilon_{1}(x, 0)\right|,\left|\Upsilon_{2}(x, 0)\right|\right\}\right\} \\
& \quad \pm u(x, 0) \geq 0, \\
& \Xi^{ \pm}(0, t)=\left(\vartheta^{*}\right)^{-1}\|\eta\| \\
& \quad+\max \left\{\left|u_{0}(0)\right|, \max \left\{\left|\Upsilon_{1}(0, t)\right|,\left|\Upsilon_{2}(0, t)\right|\right\}\right\} \\
& \quad \pm u(0, t) \geq 0, \\
& \quad \Xi^{ \pm}(1, t)=\left(\vartheta^{*}\right)^{-1}\|\eta\| \\
& \quad+\max \left\{\left|u_{0}(1)\right|, \max \left\{\left|\Upsilon_{1}(1, t)\right|,\left|\Upsilon_{2}(1, t)\right|\right\}\right\} \\
& \quad \pm u(1, t) \geq 0,
\end{aligned}
$$

Using $L_{\varepsilon}$ operator in Eq. (3) on $\Xi^{ \pm}(x, t)$ we have

$$
\begin{aligned}
& L_{\varepsilon} \Xi^{ \pm}(x, t)=\frac{\partial \Xi^{ \pm}(x, t)}{\partial t}-\varepsilon^{2} \frac{\partial^{2} \Xi^{ \pm}(x, t)}{\partial x^{2}} \\
& \quad+\theta(x) \frac{\partial \Xi^{ \pm}(x, t)}{\partial x}+\vartheta(x) \Xi^{ \pm}(x, t), \\
& =\vartheta(x)\left(\left(\vartheta^{*}\right)^{-1}\|\eta\|\right)+ \\
& \vartheta(x)\left(\max \left\{\left|u_{0}(x)\right|, \max \left\{\left|\Upsilon_{1}(x, t)\right|,\left|\Upsilon_{2}(x, t)\right|\right\}\right\}\right) \\
& \quad \pm L_{\varepsilon} u(x, t), \\
& \geq \vartheta(x)\left(\left(\vartheta^{*}\right)^{-1}\|\eta\|\right)+ \\
& \vartheta(x)\left(\max \left\{\left|u_{0}(x)\right|, \max \left\{\left|\Upsilon_{1}(x, t)\right|,\left|\Upsilon_{2}(x, t)\right|\right\}\right\}\right) \\
& \quad \pm \eta(x, t), \\
& \geq \vartheta(x)\left(\max \left\{\left|u_{0}(x)\right|, \max \left\{\left|\Upsilon_{1}(x, t)\right|,\left|\Upsilon_{2}(x, t)\right|\right\}\right\}\right) \\
& \quad+\vartheta(x)\left(\vartheta^{*}\right)^{-1}\|\eta\| \pm \eta(x, t) .
\end{aligned}
$$

Using the fact $\vartheta(x) \geq \vartheta^{*}>0$, we have $\vartheta(x)\left(\vartheta^{*}\right)^{-1} \geq 1$ and substituting it in the above inequality, we obtain

$L_{\varepsilon} \Xi^{ \pm}(x, t) \geq 0, \quad \forall(x, t) \in \bar{D}$, since $\|\eta\| \geq \eta(x, t)$.

This implies that $L_{\varepsilon} \Xi^{ \pm}(x, t) \geq 0$. Hence by Lemma (1) we have, $\Xi^{ \pm}(x, t) \geq 0, \forall(x, t) \in \bar{D}$, which gives

$$
\begin{aligned}
\|u\| & \leq\left(\vartheta^{*}\right)^{-1}\|\eta\|+ \\
& \max \left\{\left|u_{0}(x)\right|, \max \left\{\left|\Upsilon_{1}(x, t)\right|,\left|\Upsilon_{2}(x, t)\right|\right\}\right\}
\end{aligned}
$$




\section{DESCRIPTIONOF THE NUMERICAL SCHEME}

\section{A. Temporal Discretization}

We divide the time domain $[0, T]$ into $M$ sub-intervals with uniform time step size $\Delta t=\frac{T}{M}$, such that

$$
D_{\Delta t}^{M}=\left\{\left(x, t_{j}\right): x \in \Omega_{x}, t_{j}=j \Delta t, j=0(1) M\right\} .
$$

Applying the implicit Euler scheme on $t$ yields

$$
\left\{\begin{array}{l}
\left(I+\Delta t L_{\varepsilon}^{M}\right) U^{j+1}(x)=\Delta t \eta^{j+1}(x)+U^{j}(x), \\
u(x, 0)=U_{0}(x), x \in \bar{\Omega}_{x}, \\
U^{j+1}(0)=\Upsilon_{1}^{j+1}(0), j=0(1) M-1, \\
U^{j+1}(1)=\Upsilon_{2}^{j+1}(1), j=0(1) M-1 .
\end{array}\right.
$$

where,

$$
\begin{aligned}
L_{\varepsilon}^{M} U^{j+1}(x)=- & \varepsilon^{2} \frac{d^{2} U^{j+1}(x)}{d x^{2}}+\theta(x) \frac{d U^{j+1}(x)}{d x} \\
& +\vartheta(x) U^{j+1}(x) .
\end{aligned}
$$

The following lemmas show the discrete maximum principle and stability of the solution of Eq. (16).

Lemma 3 (Discrete Maximum Principle). Let $\Xi^{j+1}(x) \in C^{2}\left(\bar{\Omega}_{x}\right)$. If $\Xi^{j+1}(0) \geq 0, \Xi^{j+1}(1) \geq$ 0 , and $\left(I+\Delta t L_{\varepsilon}^{M}\right) \Xi^{j+1}(x) \geq 0, \forall x \in \Omega_{x}$, then $\Xi^{j+1}(x) \geq 0, \forall x \in \bar{\Omega}_{x}$.

Proof: Let $\left(x^{*}, t_{j+1}\right) \in\left\{\left(x, t_{j+1}\right): x \in \bar{\Omega}_{x}\right\}$ be such that

$$
\Xi^{j+1}\left(x^{*}\right)=\min _{x \in \bar{\Omega}_{x}} \Xi^{j+1}(x),
$$

and suppose that $\Xi^{j+1}\left(x^{*}\right)<0$, then we have $\left(x^{*}, t_{j+1}\right) \notin\left\{\left(0, t_{j+1}\right),\left(1, t_{j+1}\right)\right\}$. Also we have $\frac{d \Xi^{j+1}\left(x^{*}\right)}{d x}=0$, and $\frac{d^{2} \Xi^{j+1}\left(x^{*}\right)}{d x^{2}} \geq 0$. Then

$$
\begin{array}{r}
\left(I+\Delta t L_{\varepsilon}^{M}\right) \Xi^{j+1}\left(x^{*}\right)=-\varepsilon^{2} \frac{d^{2} \Xi^{j+1}\left(x^{*}\right)}{d x^{2}}+ \\
\theta\left(x^{*}\right) \frac{d \Xi^{j+1}\left(x^{*}\right)}{d x}+\vartheta\left(x^{*}\right) \Xi^{j+1}\left(x^{*}\right)<0,
\end{array}
$$

which contradicts our assumption. It follows that $\Xi^{j+1}\left(x^{*}\right) \geq 0$ and hence $\Xi^{j+1}(x) \geq 0, \forall x \in \bar{\Omega}_{x}$.

Lemma 4 Suppose $\left|\frac{\partial^{k} u(x, t)}{\partial t^{k}}\right| \leq C, \forall(x, t) \in$ $(\bar{D}), k=0,1,2$, then the local error estimate $e_{j+1}$ in the temporal direction at $(j+1)$ th time level satisfies

$$
\left\|e_{j+1}\right\| \leq C(\Delta t)^{2},
$$

where $e_{j+1}=u\left(x, t_{j+1}\right)-U^{j+1}(x)$.
Proof: The detailed proof of this lemma is given in [12]

Lemma 5 The global error estimate $E_{j}$ in the temporal direction of Eq. (16) satisfies

$$
\left\|E_{j}\right\|_{\infty} \leq C(\Delta t), \forall j \leq T / \Delta t .
$$

Proof: From Lemma 4, it follows that

$$
\begin{aligned}
& \left\|E_{j}\right\|_{\infty}=\left\|\sum_{k=1}^{j}\right\|_{\infty} \\
& \quad \leq\left\|e_{1}\right\|_{\infty}+\left\|e_{1}\right\|_{\infty}+\cdots+\left\|e_{j}\right\|_{\infty} \leq C(\Delta t) .
\end{aligned}
$$

Theorem 6 The semi-discretize solution $U^{j+1}(x)$ and its derivatives satisfy the following bounds:

$$
\begin{gathered}
\left|\frac{d^{k} U^{j+1}(x)}{d x^{k}}\right| \leq C\left(1+\left(\varepsilon^{2}\right)^{-k} \exp \left(-\theta^{*} \frac{(1-x)}{\varepsilon^{2}}\right)\right), \\
\text { for } 0 \leq k \leq 4 .
\end{gathered}
$$

Proof: The detailed proof of this theorem is given in [8].

To get the strong derivatives bound of the solution of Eq. (16), we decompose the solution into the regular and singular parts as

$$
U^{j+1}(x)=V^{j+1}(x)+W^{j+1}(x),
$$

where, the regular part $V^{j+1}(x)$ is the solution of the nonhomogeneous problem

$$
\left\{\begin{array}{c}
\left(I+\Delta t L_{\varepsilon}^{M}\right) V^{j+1}(x)=\Delta t \eta^{j+1}(x)+V^{j+1}(x), \\
\forall x \in \bar{\Omega}_{x}, \quad j=0(1) M-1 \\
V^{j+1}(0)=U^{j+1}(0), \quad j=0(1) M-1, \\
V^{j+1}(1)=U^{j+1}(1), \quad j=0(1) M-1,
\end{array}\right.
$$

and the singular part $W^{j+1}(x)$ of the homogeneous problem

$$
\left\{\begin{array}{cc}
\left(I+\Delta t L_{\varepsilon}^{M}\right) W^{j+1}(x)=0, & \forall x \in \bar{\Omega}_{x}, \\
& j=0(1) M-1, \\
W^{j+1}(0)=0, & j=0(1) M-1, \\
W^{j+1}(1)=V^{j+1}(1)-U^{j+1}(1), & j=0(1) M-1 .
\end{array}\right.
$$

Lemma 7 [9].The regular and singular components of $U^{j+1}(x)$ satisfies the following bounds:

$$
\begin{gathered}
\left|\frac{d^{k} V^{j+1}(x)}{d x^{k}}\right| \leq C\left(1+\left(\varepsilon^{2}\right)^{2-k}\right), \\
\left|\frac{d^{k} W^{j+1}(x)}{d x^{k}}\right| \leq C\left(\left(\varepsilon^{2}\right)^{-k} \exp \left(-\theta^{*} \frac{(1-x)}{\varepsilon^{2}}\right)\right), \\
\text { for } 0 \leq k \leq 3 .
\end{gathered}
$$




\section{B. Spatial discretization Mesh SelectionStrategy}

Since the boundary value problem (16) exhibits a strong boundary layer at $x=1$, we choose a piecewise-uniform Shishkin mesh on $\bar{\Omega}_{x}=[0,1]$. For this we divide the interval $[0,1]$ into two subintervals, namely $[0,1-\tau]$, and $1-\tau, 1]$, where the transition parameter $\tau$ is defined as:

$$
\tau=\min \left(\frac{1}{2}, \tau_{0} \varepsilon^{2} \ln (N)\right), \quad \tau_{0} \geq \frac{1}{\theta^{*}} .
$$

The mesh $\bar{\Omega}_{x}$ is given by:

$$
x_{i}=\left\{\begin{array}{l}
i h_{i}, \quad \text { for } i=0(1) N / 2, \\
1-\tau+\left(i-\frac{N}{2}\right) h_{i}, \text { for } i=N / 2(1) N .
\end{array}\right.
$$

where,

$$
h_{i}=x_{i}-x_{i-1}= \begin{cases}\frac{2(1-\tau)}{N}, & \text { for } i=1(1) N / 2, \\ \frac{2 \tau}{N}, & \text { for } i=N / 2+1(1) N .\end{cases}
$$

\section{B.1 Hybrid Scheme}

In this subsection, we approximate Eq. (16) by using a hybrid numerical scheme which is based on the midpoint upwind difference scheme in the outside layer regions (coarse mesh regions) and cubic spline scheme in the inside layer regions (fine mesh regions).

Let us rewrite Eq. (16) as

$$
\left\{\begin{array}{l}
L_{\varepsilon}^{M} U^{j+1}(x)=\gamma^{j+1}(x), x \in \bar{\Omega}_{x}, \quad 0(1) M-1 \\
U^{0}(x)=u_{0}(x), \quad x \in \bar{\Omega}_{x}, \\
U^{j+1}(0)=\Upsilon_{1}^{j+1}(0), \quad j=0(1) M-1, \\
U^{j+1}(1)=\Upsilon_{2}^{j+1}(1), \quad j=0(1) M-1,,
\end{array}\right.
$$

where

$$
\begin{aligned}
& L_{\varepsilon}^{M} U_{i}^{j+1}=- \\
& \quad \varepsilon^{2} \frac{d^{2} U^{j+1}(x)}{d x^{2}}+\theta(x) \frac{d U^{j+1}(x)}{d x} \\
& +Q(x) U^{j+1}(x), \\
& Q(x)=\vartheta(x)+\frac{1}{\Delta t}, \gamma^{j+1}(x)=\eta^{j+1}(x)+\frac{U^{j}(x)}{\Delta t} .
\end{aligned}
$$

\section{Midpoint upwind scheme}

The midpoint upwind method for Eq. (32) takes the form:

$$
L_{m u}^{N, M} U_{i}^{j+1}=\left\{\begin{array}{l}
-\varepsilon^{2} D_{x}^{+} D_{x}^{-} U_{i}^{j+1}+\theta_{i-1 / 2} D_{x}^{-} U_{i}^{j+1} \\
+Q_{i-1 / 2} U_{i-1 / 2}^{j+1}=\gamma_{i-1 / 2}^{j+1} \\
U^{j+1}(0)=\Upsilon^{j+1}(0), \quad 0 \leq j \leq T \\
U^{j+1}(1)=\Upsilon^{j+1}(1), \quad 0 \leq j \leq T .
\end{array}\right.
$$

where,

$$
\begin{aligned}
& D_{x}^{-} U_{i}^{j+1}=\frac{U_{i}^{j+1}-U_{i-1}^{j+1}}{h_{i}}, \\
& D_{x}^{+} D_{x}^{-} U_{i, j+1}=\frac{2}{h_{i}+h_{i-1}}\left(\frac{U_{i+1}^{j+1}-U_{i}^{j+1}}{h_{i}}-\frac{U_{i}^{j+1}-U_{i-1}^{j+1}}{h_{i-1}}\right), \\
& \theta_{i-1 / 2}=\left(\frac{\theta_{i}+\theta_{i-1}}{2}\right), Q_{i-1 / 2}=\left(\frac{Q_{i}+Q_{i-1}}{2}\right), \text { and } \\
& \gamma_{i-1 / 2}^{j+1}=\left(\frac{\gamma_{i}^{j+1}+\gamma_{i-1}^{j+1}}{2}\right) .
\end{aligned}
$$

and $L_{m u}^{N, M} U_{i}^{j+1}$ is the midpoint upwind finite difference operator.

Thus, we get the following system of equations

$$
\begin{gathered}
L_{m u}^{N, M} U_{i}^{j+1}=s_{i}^{-} U_{i-1}^{j+1}+s_{i}^{0} U_{i}^{j+1}+s_{i}^{+} U_{i+1}^{j+1}=B_{i}^{j+1} \\
i=1(1) N-1, j=0(1) M-1 .
\end{gathered}
$$

where

$$
\begin{aligned}
& s_{i}^{-}=\frac{-2 \varepsilon^{2}}{h_{i-1}\left(h_{i}+h_{i-1}\right)}-\frac{\theta_{i-1 / 2}}{h_{i}}+\frac{Q_{i-1 / 2}}{2}, \\
& s_{i}^{0}=\frac{2 \varepsilon^{2}}{h_{i} h_{i-1}}+\frac{\theta_{i-1 / 2}}{h_{i}}+\frac{Q_{i-1 / 2}}{2}, \\
& s_{i}^{+}=\frac{-2 \varepsilon^{2}}{h_{i}\left(h_{i}+h_{i-1}\right)}, \\
& B_{i}^{j+1}=\gamma_{i-1 / 2}^{j+1} .
\end{aligned}
$$

\section{Cubic spline difference scheme}

Now, we approximate the inside layer region of the resulting spatial Eq. (32) by applying the cubic spline method as described below. An interpolating cubic spline function $S^{j+1}(x)$ corresponding to the values $U^{j+1}\left(x_{0}\right), U^{j+1}\left(x_{1}\right), U^{j+1}\left(x_{2}\right), \cdots, U^{j+1}\left(x_{N}\right)$, of a function $U^{j+1}(x)$ at the points $x_{0}, x_{1}, x_{2}, \cdots, x_{N}$ and it satisfies the following properties:

1. $S^{j+1}(x)$ coincides with a polynomial of degree three on each subintervals $\left[x_{i-1}, x_{i}\right], i=1(1) N$,

2. $S^{j+1}(x) \in C^{2}(\bar{D})$,

3. $S^{j+1}\left(x_{i}\right)=U^{j+1}\left(x_{i}\right)$.

Now, for $x \in\left[x_{i-1}, x_{i}\right]$, the cubic spline function $S^{j+1}(x)$ can be written as [17]:

$$
\begin{gathered}
S^{j+1}(x)=\frac{\left(x_{i}-x\right)^{3}}{6 h_{i}} M_{i-1}+\frac{\left(x-x_{i-1}\right)^{3}}{6 h_{i}} M_{i} \\
+\left(U_{i-1}^{j+1}-\frac{h_{i}^{2}}{6} M_{i-1}\right)\left(\frac{x_{i}-x}{h_{i}}\right) \\
+\left(U_{i}^{j+1}-\frac{h_{i}^{2}}{6} M_{i}\right)\left(\frac{x-x_{i-1}}{h_{i}}\right), \\
x \in\left[x_{i-1}, x_{i}\right], \quad i=1(1) N .
\end{gathered}
$$

where, $M_{i}=\frac{d^{2} S^{j+1}\left(x_{i}\right)}{d x^{2}}, i=1(1) N$.

From the properties of cubic spline [18], we obtain,

$$
\begin{aligned}
& \frac{h_{i}}{6} M_{i-1}+\frac{h_{i}+h_{i+1}}{3} M_{i}+\frac{h_{i+1}}{6} M_{i+1} \\
& =\frac{U_{i+1, j}-U_{i, j+1}}{h_{i+1}}-\frac{U_{i, j+1}-U_{i-1, j+1}}{h_{i}}, \\
& \quad i=1(1) N-1, j=1(1) M-1 .
\end{aligned}
$$


Using Taylor's series approximations for $U^{j+1}\left(x_{k}\right), k=i \pm 1$ in the spatial variable, we have:

$$
\begin{aligned}
& U^{j+1}\left(x_{i-1}\right) \approx \\
& U^{j+1}\left(x_{i}\right)-h_{i-1} \frac{d U^{j+1}\left(x_{i}\right)}{d x}+\frac{h_{i-1}^{2}}{2} \frac{d^{2} U^{j+1}\left(x_{i}\right)}{d x^{2}}, \\
& \begin{aligned}
& U^{j+1}\left(x_{i+1}\right) \approx \\
& U^{j+1}\left(x_{i}\right)+h_{i} \frac{d U^{j+1}\left(x_{i}\right)}{d x}+\frac{h_{i}^{2}}{2} \frac{d^{2} U^{j+1}\left(x_{i}\right)}{d x^{2}} .
\end{aligned}
\end{aligned}
$$

Multiplying Eq. (40) by $h_{i}^{2} / h_{i-1}^{2}$ and then subtracting the resulting equations from Eq. (41), we have:

$$
\begin{aligned}
& \frac{d U^{j+1}\left(x_{i}\right)}{d x} \approx \\
& \frac{\left(-h_{i}^{2} U^{j+1}\left(x_{i-1}\right)+\left(h_{i}^{2}-h_{i-1}^{2}\right) U^{j+1}\left(x_{i}\right)+h_{i-1}^{2} U^{j+1}\left(x_{i+1}\right)\right)}{h_{i} h_{i-1}\left(h_{i}+h_{i-1}\right)}
\end{aligned}
$$

Similarly, multiplying (40) by $h_{i} / h_{i-1}$ and then adding the resulting equations to Eq. (41), we get:

$$
\begin{aligned}
& \frac{d^{2} U^{j+1}\left(x_{i}\right)}{d x^{2}} \approx \\
& \frac{2\left(h_{i} U^{j+1}\left(x_{i-1}\right)+\left(h_{i}+h_{i-1}\right) U^{j+1}\left(x_{i}\right)+h_{i-1} U^{j+1}\left(x_{i+1}\right)\right)}{h_{i} h_{i-1}\left(h_{i}+h_{i-1}\right)}
\end{aligned}
$$

Inserting Eqs. (42) and (43) in $\frac{d U^{j+1}\left(x_{i+1}\right)}{d x} \approx$ $\frac{d U^{j+1}\left(x_{i}\right)}{d x}+h_{i} \frac{d^{2} U^{j+1}\left(x_{i+1}\right)}{d x^{2}}$ and $\frac{d U^{j+1}\left(x_{i-1}\right)}{d x} \approx$ $\frac{d U^{j+1}\left(x_{i}\right)}{d x}+h_{i-1} \frac{d^{2} U^{j+1}\left(x_{i+1}\right)}{d x^{2}}$, we obtain

$$
\begin{array}{r}
\frac{d U^{j+1}\left(x_{i-1}\right)}{d x} \approx \frac{\left(-\left(h_{i}^{2}+2 h_{i} h_{i-1}\right) U^{j+1}\left(x_{i-1}\right)\right)}{h_{i} h_{i-1}\left(h_{i}+h_{i-1}\right)}+ \\
\frac{\left(\left(h_{i}+h_{i-1}\right)^{2} U^{j+1}\left(x_{i}\right)-h_{i-1}^{2} U^{j+1}\left(x_{i+1}\right)\right)}{h_{i} h_{i-1}\left(h_{i}+h_{i-1}\right)}
\end{array}
$$

$$
\begin{aligned}
& \frac{d U^{j+1}\left(x_{i+1}\right)}{d x} \approx \frac{\left(-h_{i}^{2} U^{j+1}\left(x_{i-1}\right)-\left(h_{i}+h_{i-1}\right)^{2} U^{j+1}\left(x_{i}\right)\right)}{h_{i} h_{i-1}\left(h_{i}+h_{i-1}\right)} \\
&+\frac{\left(\left(h_{i}^{2}+2 h_{i} h_{i-1}\right) U^{j+1}\left(x_{i+1}\right)\right)}{h_{i} h_{i-1}\left(h_{i}+h_{i-1}\right)}
\end{aligned}
$$

Equation (32) at $x=x_{k}, k=i, i \pm 1$ can be written as:

$$
\varepsilon^{2} M_{k}=\theta_{k} \frac{d U^{j+1}\left(x_{k}\right)}{d x}+Q_{k} U^{j+1}\left(x_{k}\right)-\gamma^{j+1}\left(x_{k}\right) .
$$

We have,

Substituting Eqs. (42) - (45) into Eq. (46) and using the resulting equation into Eq. (39) yields

$$
\begin{gathered}
L_{c s}^{N, M}=r_{i}^{-} U_{i-1}^{j+1}+r_{i}^{0} U_{i}^{j+1}+r_{i}^{+} U_{i+1}^{j+1}=F_{i}^{j+1}, \\
i=1(1) N-1, j=0(1) M-1 .
\end{gathered}
$$

where, $L_{c s}^{N}$ is the cubic spline operator,

$$
\begin{aligned}
r_{i}^{-}= & -\frac{\theta_{i-1}\left(h_{i}+2 h_{i-1}\right)}{2\left(h_{i-1}+h_{i}\right)}+\frac{h_{i-1} Q_{i-1}}{2}-\frac{h_{i} \theta_{i}}{h_{i-1}} \\
& +\frac{\theta_{i+1} h_{i}^{2}}{2 h_{i-1}\left(h_{1-1}+h_{i}\right)}-\frac{3 \varepsilon^{2}}{h_{i-1}}, \\
r_{i}^{0}= & \frac{\theta_{i-1}\left(h_{i-1}+h_{i}\right)}{2 h_{i}}+\frac{\theta_{i}\left(h_{i}^{2}-h_{i-1}^{2}\right)}{h_{i} h_{i-1}}+\left(h_{i}+h_{i-1}\right) Q_{i} \\
& -\frac{\theta_{i+1}\left(h_{i-1}+h_{i}\right)}{2 h_{i-1}}+\frac{3 \varepsilon^{2}\left(h_{i}+h_{i-1}\right)}{h_{i} h_{h_{i-1}}}, \\
r_{i}^{+}=- & \frac{\theta_{i-1} h_{i-1}^{2}}{2 h_{i}\left(h_{i-1}+h_{i}\right)}+\frac{h_{i} Q_{i+1}}{2}+\frac{h_{i-1} \theta_{i}}{h_{i}} \\
& \quad+\frac{\theta_{i+1}\left(h_{i-1}+2 h_{i}\right)}{2\left(h_{i}+h_{i-1}\right)}-\frac{3 \varepsilon^{2}}{h_{i}} \\
F_{i}^{j+1}= & \frac{h_{i-1}}{2} \gamma_{i-1}^{j+1}+\left(h_{i}+h_{i-1}\right) \gamma_{i}^{j+1}+\frac{h_{i}}{2} \gamma_{i+1}^{j+1} .
\end{aligned}
$$

The total discrete scheme takes the form:

$$
L_{h y b}^{N, M} U_{i}^{j+1}=\left\{\begin{array}{l}
L_{m u}^{N, M} U_{i}^{j+1}=\gamma_{i-1 / 2, j+1}, \\
L_{c s}^{N, M} U_{i}^{j+1}=\gamma_{i}^{j+1}, \\
U_{0}^{j+1}=\Upsilon_{1}^{j+1}(0), \\
U_{N}^{j+1}=\Upsilon_{2}^{j+1}(1), \\
U_{i, 0}=U\left(x_{i}, 0\right) .
\end{array}\right.
$$

Thus, we obtain the system of linear equations as:

$$
\begin{gathered}
R_{i}^{-} U_{i-1}^{j+1}+R_{i}^{0} U_{i}^{j+1}+R_{i}^{+} U_{i+1}^{j+1}=D_{i}^{j+1}, \\
i=1(1) N-1, j=0(1) M-1 .
\end{gathered}
$$

where,

$$
\begin{aligned}
& R_{i}^{-}=\left\{\begin{array}{l}
\frac{-2 \varepsilon^{2}}{h_{i-1}\left(h_{i}+h_{i-1}\right)}-\frac{\theta_{i-1 / 2}}{h_{i}}+\frac{Q_{i-1 / 2}}{2}, i=1(1) N / 2, \\
\frac{\theta_{i-1}\left(h_{i}+2 h_{i-1}\right)}{2\left(h_{i-1}+h_{i}\right)}+\frac{h_{i-1} Q_{i-1}}{2}-\frac{h_{i} \theta_{i}}{h_{i-1}} \\
+\frac{\theta_{i+1} h_{i}^{2}}{2 h_{i-1}\left(h_{1-1}+h_{i}\right)}-\frac{3 \varepsilon^{2}}{h_{i-1}}, i=N / 2+1(1) N-1 .
\end{array}\right. \\
& R_{i}^{0}=\left\{\begin{array}{l}
\frac{2 \varepsilon^{2}}{h_{i} h_{i-1}}+\frac{\theta_{i-1 / 2}}{h_{i}}+\frac{Q_{i-1 / 2}}{2}, i=1(1) N / 2, \\
\frac{\theta_{i-1}\left(h_{i-1}+h_{i}\right)}{2 h_{i}}+\frac{\theta_{i}\left(h_{i}^{2}-h_{i-1}^{2}\right)}{h_{i} h_{i-1}}+\left(h_{i}+h_{i-1}\right) Q_{i} \\
-\frac{\theta_{i+1}\left(h_{i-1}+h_{i}\right)}{2 h_{i-1}}+\frac{3 \varepsilon^{2}\left(h_{i}+h_{i-1}\right)}{h_{i} h_{h_{i-1}}}, i=N / 2+1(1) N-1 .
\end{array}\right. \\
& R_{i}^{+}=\left\{\begin{array}{l}
\frac{-2 \varepsilon^{2}}{h_{i}\left(h_{i}+h_{i-1}\right)}, \quad 0<i \leq N / 2, \\
-\frac{\theta_{i-1} h_{i-1}^{2}}{2 h_{i}\left(\left(h_{i-1}+h_{i}\right)\right)}+\frac{h_{i} Q_{i+1}}{2}+\frac{h_{i-1} \theta_{i}}{h_{i}} \\
+\frac{\theta_{i+1}\left(h_{i-1}+2 h_{i}\right)}{2\left(h_{i}+h_{i-1}\right)}-\frac{3 \varepsilon^{2}}{h_{i}}, i=N / 2+1(1) N .
\end{array}\right. \\
& D_{i}^{j+1}=\left\{\begin{array}{l}
B_{i}^{j+1}, \quad i=0(i) N / 2,0(1) M-1 \\
F_{i}^{j+1}, i=N / 2+1(1) N-1,0(1) M-1 .
\end{array}\right.
\end{aligned}
$$

Lemma 8 (Discrete Maximum Principle). If $\Xi_{0}^{j+1} \geq$ $0, \Xi_{1}^{j+1} \geq 0$, then $L_{\varepsilon}^{N, M} \Xi_{i}^{j+1} \geq 0, \forall i=1(1), N-1$ implies $\Xi_{i}^{j+1} \geq 0, \forall i=0(1) N$.

Proof: The proof follows from the similar arguments as used in Lemma 3. 
Lemma 9 The solution $U_{i}^{j+1}$ of Eq. (49) at $(j+1)$ th time level and $Q\left(x_{i}\right) \geq \sigma>0$ holds

$$
\begin{aligned}
& \left\|U_{i}^{j+1}\right\| \leq \frac{\|\gamma\|}{\sigma} \\
& +C \max \left\{\left\|\Upsilon_{1}^{j+1}\left(x_{i}\right)\right\|\right\},\left\|\Upsilon_{2}^{j+1}\left(x_{i}\right)\right\|, i=0(1) \cdots N .
\end{aligned}
$$

Proof: By considering the barrier functions

$$
\begin{aligned}
& \left(\Xi^{ \pm}\right)_{i}^{j+1}=\frac{\|\gamma\|}{\sigma} \\
& \quad+C \max \left\{\left\|\Upsilon_{1}^{j+1}\left(x_{i}\right)\right\|\right\},\left\|\Upsilon_{2}^{j+1}\left(x_{i}\right)\right\| \pm U_{i}^{j+1}
\end{aligned}
$$

and applying Lemma 8 we obtain the required bound.

\section{ERROR ANALYSIS}

To establish an $\varepsilon$-uniform convergence of the discrete scheme (49), we decompose the obtained solution into regular and singular parts as

$$
U_{i, j+1}=V_{i, j+1}+W_{i, j+1}
$$

where the regular part is

$$
\left\{\begin{array}{l}
L_{h y b}^{N, M} V\left(x_{i}, t_{j+1}\right)=\left\{\begin{array}{l}
\gamma\left(x_{i}, t_{j+1}\right), \\
0(1) N / 2,0(1) M-1 \\
\gamma\left(x_{i-1 / 2}, t_{j+1}\right), \\
N / 2+1(1) N, 0(1) M-1
\end{array}\right. \\
V\left(0, t_{j+1}\right)=V\left(0, t_{j+1}\right), 0<j \leq M-1, \\
V\left(1, t_{j+1}\right)=V\left(1, t_{j+1}\right), 0<j \leq M-1 \\
. V(i, 0)=V\left(x_{i}, 0\right), 0<j \leq M-1 \\
.
\end{array}\right.
$$

and the singular part is

$$
\left\{\begin{array}{l}
L_{h y b}^{N, M} W\left(x_{i}, t_{j+1}\right)=0,0(1) N, 0(1) M-1, \\
W(0, j+1)=W\left(0, t_{j+1}\right), 0(1) M-1, \\
W(1, j+1)=W\left(1, t_{j+1}\right), 0(1) M-1 \\
W(i, 0)=W\left(x_{i}, 0\right), \quad 0(1) N
\end{array}\right.
$$

Lemma 10 The regular component satisfies the following estimate at each mesh point $\left(x_{i}, t_{j+1}\right) \in \bar{D}$

$$
\left|(v-V)\left(x_{i}, t_{j+1}\right)\right| \leq C\left(\Delta t+N^{-1}\left(\varepsilon^{2}+N^{-1}\right)\right)
$$

Proof: From the truncation error estimate, we have

$$
\begin{aligned}
& \left|L_{h y b}^{N, M}(v-V)\left(x_{i}, t_{j+1}\right)\right| \\
& \leq C\left(\Delta t+\varepsilon^{2} \int_{x_{i-1}}^{x_{i+1}}\left|v^{\prime \prime \prime}\left(\omega, t_{j+1}\right)\right| d \omega\right) \\
& +C\left(h_{i} \int_{x_{i-1}}^{x_{i+1}}\left|v^{\prime \prime \prime}\left(\omega, t_{j+1}\right)\right| d \omega\right) \\
& \leq C\left(\Delta t+\left(h_{i+1}+h_{i}\right) \varepsilon^{2}+h_{i}^{2}\right)
\end{aligned}
$$

Let $H=\max \left\{h_{i}\right\}$. Then, we have

$$
\left|L_{h y b}^{N, M}(v-V)\left(x_{i}, t_{j+1}\right)\right| \leq C\left(\Delta t+H\left(\varepsilon^{2}+H\right)\right)
$$

Let us consider the barrier function for $i=1(1) N-$ $1, j \Delta t \leq T \beta^{ \pm}\left(x_{i}, t_{j+1}\right)=C\left(\Delta t+H\left(\varepsilon^{2}+H\right)\right) \pm$ $(v-V)\left(x_{i}, t_{j+1}\right)$. Clearly, we have $\beta^{ \pm}\left(x_{0}, t_{j+1}\right) \geq 0$, $\beta^{ \pm}\left(x_{N}, t_{j+1}\right) \geq 0$ and $L_{h y b}^{N, M} \beta^{ \pm}\left(x_{i}, t_{j+1}\right) \geq 0$.

Thus, an application of discrete maximum principle gives

$$
\left|(v-V)\left(x_{i}, t_{j+1}\right)\right| \leq C\left(\Delta t+N^{-1}\left(\varepsilon^{2}+N^{-1}\right)\right) .
$$

Lemma 11 The singular component satisfies the following estimate at each mesh point $\left(x_{i}, t_{j+1}\right) \in \bar{D}$ :

$$
\left|(w-W)\left(x_{i}, t_{j+1}\right)\right| \leq C\left(\Delta t+N^{-2}(\ln N)^{2}\right) .
$$

Proof: From the truncation error estimate, we have

$$
\begin{gathered}
\left|L_{h y b}^{N, M}(w-W)\left(x_{i}, t_{j+1}\right)\right| \leq \varepsilon^{2} h_{i}^{2}\left\|w^{(4)}\left(x_{i}, t_{j+1}\right)\right\| \\
\leq C\left(\Delta t+N^{-2}(\ln N)^{2}\right) .
\end{gathered}
$$

By considering the barrier function for $i=1(1) N-$ $1, j \Delta t \leq T$

$$
\begin{aligned}
\beta^{ \pm}\left(x_{i}, t_{j+1}\right) & =C\left(\Delta t+N^{-2}(\ln N)^{2}\right) \\
& \pm\left|(w-W)\left(x_{i}, t_{j+1}\right)\right|
\end{aligned}
$$

we obtain $\beta^{ \pm}\left(x_{0}, t_{j+1}\right) \geq 0, \beta^{ \pm}\left(x_{N}, t_{j+1}\right) \geq 0$ and $L_{h y b}^{N, M} \beta^{ \pm}\left(x_{i}, t_{j+1}\right) \geq 0$. Therefore, an application of discrete maximum principle gives

$$
\left|(w-W)\left(x_{i}, t_{j+1}\right)\right| \leq C\left(\Delta t+N^{-2}(\ln N)^{2}\right)
$$

Theorem 12 Let $u(x, t)$ be the solution of the problem (1) and $U(x, t)$ be the numerical solution of the corresponding discrete problem (49), then at each mesh point $\left(x_{i}, t_{j+1}\right) \in \bar{D}$, then we have

$$
\left|(u-U)\left(x_{i}, t_{j+1}\right)\right| \leq C\left(\Delta t+N^{-2}(\ln N)^{2}\right) .
$$

Proof: From the triangular inequality, we have

$$
\begin{aligned}
\left|(u-U)\left(x_{i}, t_{j+1}\right)\right| & \leq\left|w\left(x_{i}, t_{j+1}\right)-W\left(x_{i}, t_{j+1}\right)\right| \\
+ & \left|v\left(x_{i}, t_{j+1}\right)-V\left(x_{i}, t_{j+1}\right)\right|
\end{aligned}
$$

From Lemma 10 and Lemma 11, we get the desired result. 


\section{NUMERICAL EXAMPLES AND RESULTS}

To verify the efficiency of the proposed scheme for the problem (1), some model examples are presented. As the exact solutions of these examples are not known, the maximum pointwise error for the given examples is computed by using the double mesh principle [19] defined by:

$$
E_{\varepsilon, \delta, \eta}^{N, M}=\max _{1 \leq i, j \leq N-1, M-1}\left|U_{i, j}^{N, M}-U_{i, j}^{2 N, 2 M}\right|
$$

where $U_{i, j}^{N, M}$ and $U_{i, j}^{2 N, 2 M}$ are the computed numerical solutions obtained on the mesh $D^{N, M}=\Omega_{x}^{N} \times \Omega_{t}^{M}$ and $D^{2 N, 2 M}=\Omega_{x}^{2 N} \times \Omega_{t}^{2 M}$ respectively. $N$ and $M$ are mesh intervals in the spatial direction temporal direction respectively.

For any value of $N$ and $M$ the $\varepsilon$-uniform errors $\left(E^{N, M}\right)$, rate of convergence $\left(r_{\varepsilon, \delta, \eta}^{N, M}\right)$ and $\varepsilon$-uniform convergence $\left(r^{N, M}\right)$ are calculated by the following formula

$$
\begin{aligned}
& E^{N, M}=\max _{\varepsilon, \delta, \eta} E_{\varepsilon, \delta, \eta}^{N, M}, \quad r_{\varepsilon, \delta, \eta}^{N, M}=\log _{2}\left(\frac{E_{\varepsilon, \delta, \eta}^{N, M}}{E_{\varepsilon, \delta, \eta}^{2 N, 2 M}}\right) \\
& \text { and } r^{N, M}=\log _{2}\left(\frac{E^{N, M}}{E^{2 N, 2 M}}\right) .
\end{aligned}
$$

respectively.

Example 13 [9] We consider the following SPPPDDE of the form in (1)

$$
\left\{\begin{array}{l}
\frac{\partial u}{\partial t}-\varepsilon^{2} \frac{\partial^{2} u}{\partial x^{2}}+\left(2+x+x^{2}\right) \frac{\partial u}{\partial x}+\left(\frac{1+x^{2}}{2}\right) u(x-\delta, t) \\
=\sin (\pi x(1-x)) \\
u_{0}(x)=0, \Upsilon_{1}(0, t)=\Upsilon_{2}(1, t)=0, T=1
\end{array}\right.
$$

Example 14 [13] Now we consider the following SPPPDDE of the form in (1)

$$
\left\{\begin{array}{l}
\frac{\partial u}{\partial t}-\varepsilon^{2} \frac{\partial^{2} u}{\partial x^{2}}+\left(2+x+x^{2}\right) \frac{\partial u}{\partial x}+\left(\frac{1+x^{2}}{2}\right) u(x-\delta, t) \\
=\sin (\pi x(1-x)) t \\
u_{0}(x)=0, \Upsilon_{1}(0, t)=\Upsilon_{2}(1, t)=0, T=1
\end{array}\right.
$$


Table 1: $E_{\varepsilon, \delta}^{N, M}$ for Example(13) with $T=1.0, \delta=0.5 \times \varepsilon, M=N$

\begin{tabular}{|c|c|c|c|c|c|}
\hline$\varepsilon \downarrow$ & $\mathrm{N}=32$ & $\mathrm{~N}=64$ & $\mathrm{~N}=128$ & $\mathrm{~N}=256$ & $\mathrm{~N}=512$ \\
\hline \multicolumn{6}{|c|}{ Proposed hybrid scheme } \\
\hline $10^{0}$ & $1.9998 \mathrm{e}-03$ & $1.0844 \mathrm{e}-03$ & $5.6678 \mathrm{e}-04$ & $2.8994 \mathrm{e}-04$ & $1.4670 \mathrm{e}-04$ \\
\hline $10^{-1}$ & $5.5098 \mathrm{e}-03$ & $3.3181 \mathrm{e}-03$ & $1.9023 \mathrm{e}-03$ & $1.0456 \mathrm{e}-03$ & $5.5458 \mathrm{e}-04$ \\
\hline $10^{-2}$ & $5.8712 \mathrm{e}-03$ & $3.5891 \mathrm{e}-03$ & $2.1232 \mathrm{e}-03$ & $1.2098 \mathrm{e}-03$ & $6.6900 \mathrm{e}-04$ \\
\hline $10^{-4}$ & $5.8762 \mathrm{e}-03$ & $3.5944 \mathrm{e}-03$ & $2.1278 \mathrm{e}-03$ & $1.2139 \mathrm{e}-03$ & $6.7189 \mathrm{e}-04$ \\
\hline $10^{-6}$ & $5.8762 \mathrm{e}-03$ & $3.5944 \mathrm{e}-03$ & $2.1278 \mathrm{e}-03$ & $1.2139 \mathrm{e}-03$ & $6.7189 \mathrm{e}-04$ \\
\hline $10^{-8}$ & $5.8762 \mathrm{e}-03$ & $3.5944 \mathrm{e}-03$ & $2.1278 \mathrm{e}-03$ & $1.2139 \mathrm{e}-03$ & $6.7189 \mathrm{e}-04$ \\
\hline \multicolumn{6}{|c|}{ Midpoit Upwind scheme } \\
\hline $10^{0}$ & $2.1994 \mathrm{e}-03$ & $1.1778 \mathrm{e}-03$ & $6.1092 \mathrm{e}-04$ & $3.1142 \mathrm{e}-04$ & $1.5725 \mathrm{e}-04$ \\
\hline $10^{-1}$ & $7.4106 \mathrm{e}-03$ & $4.6999 \mathrm{e}-03$ & $2.8947 \mathrm{e}-03$ & $1.7047 \mathrm{e}-03$ & $9.6838 \mathrm{e}-04$ \\
\hline $10^{-2}$ & $7.2526 \mathrm{e}-03$ & $4.6756 \mathrm{e}-03$ & $2.9359 \mathrm{e}-03$ & $1.7694 \mathrm{e}-03$ & $1.0286 \mathrm{e}-03$ \\
\hline $10^{-4}$ & $7.2470 \mathrm{e}-03$ & $4.6739 \mathrm{e}-03$ & $2.9358 \mathrm{e}-03$ & $1.7697 \mathrm{e}-03$ & $1.0295 \mathrm{e}-03$ \\
\hline $10^{-6}$ & $7.2470 \mathrm{e}-03$ & $4.6739 \mathrm{e}-03$ & $2.9358 \mathrm{e}-03$ & $1.7696 \mathrm{e}-03$ & $1.0295 \mathrm{e}-03$ \\
\hline $10^{-8}$ & $7.2470 \mathrm{e}-03$ & $4.6739 \mathrm{e}-03$ & $2.9358 \mathrm{e}-03$ & $1.7696 \mathrm{e}-03$ & $1.0295 \mathrm{e}-03$ \\
\hline \multicolumn{6}{|c|}{ Cubic Spline scheme } \\
\hline $10^{0}$ & $1.8277 \mathrm{e}-03$ & $1.0130 \mathrm{e}-03$ & $5.3456 \mathrm{e}-04$ & $2.7472 \mathrm{e}-04$ & $1.3931 \mathrm{e}-04$ \\
\hline $10^{-1}$ & $8.8650 \mathrm{e}-03$ & $4.6533 \mathrm{e}-03$ & $2.3350 \mathrm{e}-03$ & $1.1456 \mathrm{e}-03$ & $5.6062 \mathrm{e}-04$ \\
\hline $10^{-2}$ & $1.6202 \mathrm{e}-02$ & $9.0831 \mathrm{e}-03$ & $3.6470 \mathrm{e}-03$ & $1.7450 \mathrm{e}-03$ & $9.0803 \mathrm{e}-04$ \\
\hline $10^{-4}$ & $1.6741 \mathrm{e}-02$ & $1.0469 \mathrm{e}-02$ & $5.8106 \mathrm{e}-03$ & $3.0480 \mathrm{e}-03$ & $1.5582 \mathrm{e}-03$ \\
\hline $10^{-6}$ & $1.6741 \mathrm{e}-02$ & $1.0469 \mathrm{e}-02$ & $5.8109 \mathrm{e}-03$ & $3.0486 \mathrm{e}-03$ & $1.5582 \mathrm{e}-03$ \\
\hline $10^{-8}$ & $1.6741 \mathrm{e}-02$ & $1.0469 \mathrm{e}-02$ & $5.8109 \mathrm{e}-03$ & $3.0486 \mathrm{e}-03$ & $1.5582 \mathrm{e}-03$ \\
\hline \multicolumn{6}{|c|}{ Results in [9] } \\
\hline $10^{0}$ & $1.51 \mathrm{e}-03$ & $7.59 \mathrm{e}-04$ & $3.81 \mathrm{e}-04$ & $1.91 \mathrm{e}-04$ & $9.57 \mathrm{e}-05$ \\
\hline $10^{-1}$ & $6.83 e-03$ & $4.03 \mathrm{e}-03$ & $2.36 \mathrm{e}-03$ & $1.35 \mathrm{e}-03$ & 7.61e-04 \\
\hline $10^{-2}$ & $8.25 e-03$ & $4.91 \mathrm{e}-03$ & $2.91 \mathrm{e}-03$ & $1.68 \mathrm{e}-03$ & $9.38 \mathrm{e}-04$ \\
\hline $10^{-4}$ & $8.48 \mathrm{e}-03$ & $5.13 e-03$ & $3.09 \mathrm{e}-03$ & $1.81 \mathrm{e}-03$ & $1.03 e-03$ \\
\hline $10^{-6}$ & $8.48 \mathrm{e}-03$ & $5.13 e-03$ & $3.09 e-03$ & $1.81 \mathrm{e}-03$ & $1.03 e-03$ \\
\hline $10^{-8}$ & $8.48 \mathrm{e}-03$ & $5.13 e-03$ & $3.09 \mathrm{e}-03$ & $1.81 \mathrm{e}-03$ & $1.03 e-03$ \\
\hline
\end{tabular}


Table 2: $E^{N, M}$ and $r^{N, M}$ for Example (13) with $T=1.0, \delta=0.5 \times \varepsilon, M=N$

\begin{tabular}{|c|c|c|c|c|c|}
\hline$\varepsilon \downarrow$ & $\mathrm{N}=32$ & $\mathrm{~N}=64$ & $\mathrm{~N}=128$ & $\mathrm{~N}=256$ & $\mathrm{~N}=512$ \\
\hline \multicolumn{6}{|c|}{ Proposed hybrid scheme } \\
\hline$E^{N, M}$ & $5.8762 \mathrm{e}-03$ & $3.5944 \mathrm{e}-03$ & $2.1278 \mathrm{e}-03$ & $1.2139 \mathrm{e}-03$ & $6.7189 \mathrm{e}-04$ \\
\hline$r^{N, M}$ & 0.70913 & 0.75639 & 0.80971 & 0.85335 & 0.90419 \\
\hline \multicolumn{6}{|c|}{ Midpoint Upwind scheme } \\
\hline$E^{N, M}$ & $7.4106 \mathrm{e}-03$ & $4.6999 \mathrm{e}-03$ & $2.9359 \mathrm{e}-03$ & $1.7697 \mathrm{e}-03$ & $1.0295 \mathrm{e}-03$ \\
\hline$r^{N, M}$ & $6.5696 \mathrm{e}-01$ & $6.7883 \mathrm{e}-01$ & $7.3030 \mathrm{e}-01$ & 0.7816 & 0.81624 \\
\hline \multicolumn{6}{|c|}{ Cubic spline scheme } \\
\hline$E^{N, M}$ & $1.6741 \mathrm{e}-02$ & $1.0469 \mathrm{e}-02$ & $5.8109 \mathrm{e}-03$ & $3.0486 \mathrm{e}-03$ & $1.5582 \mathrm{e}-03$ \\
\hline$r^{N, M}$ & 0.67726 & 0.84929 & 0.93061 & 0.96827 & 0.98453 \\
\hline \multicolumn{6}{|c|}{ Results in [9] } \\
\hline$E^{N, M}$ & $8.48 \mathrm{e}-03$ & $5.13 \mathrm{e}-03$ & $3.09 \mathrm{e}-03$ & $1.81 \mathrm{e}-03$ & $1.03 \mathrm{E}-03$ \\
\hline$r^{N, M}$ & 0.73 & 0.73 & 0.77 & 0.81 & .84 \\
\hline
\end{tabular}


Table 3: $E_{\varepsilon, \delta}^{(N, M)}, E^{(N, M)}$ and $r^{(N, M)}$ for Example (14) with $T=1.0, \delta=0.5 \times \varepsilon, N=M$

\begin{tabular}{|c|c|c|c|c|c|}
\hline$\varepsilon \downarrow$ & $\mathrm{N}=32$ & $\mathrm{~N}=64$ & $\mathrm{~N}=128$ & $\mathrm{~N}=256$ & $\mathrm{~N}=512$ \\
\hline \multicolumn{6}{|c|}{ Proposed hybrid scheme } \\
\hline $10^{0}$ & $5.7811 \mathrm{e}-04$ & $2.9885 \mathrm{e}-04$ & $1.5194 \mathrm{e}-04$ & $7.6609 \mathrm{e}-05$ & $3.8466 \mathrm{e}-05$ \\
\hline $10^{-1}$ & $1.3530 \mathrm{e}-03$ & $7.8909 \mathrm{e}-04$ & $4.2438 \mathrm{e}-04$ & $2.1891 \mathrm{e}-04$ & $1.1047 \mathrm{e}-04$ \\
\hline $10^{-2}$ & $1.4572 \mathrm{e}-03$ & $8.6051 \mathrm{e}-04$ & $4.7185 e-04$ & $2.5755 \mathrm{e}-04$ & $1.3466 \mathrm{e}-04$ \\
\hline $10^{-4}$ & $1.4557 \mathrm{e}-03$ & $8.5972 \mathrm{e}-04$ & $4.7250 \mathrm{e}-04$ & $2.5800 \mathrm{e}-04$ & $1.3495 \mathrm{e}-04$ \\
\hline $10^{-6}$ & $1.4556 \mathrm{e}-03$ & $8.5970 \mathrm{e}-04$ & $4.7249 \mathrm{e}-04$ & $2.5799 \mathrm{e}-04$ & $1.3494 \mathrm{e}-04$ \\
\hline $10^{-8}$ & $1.4556 \mathrm{e}-03$ & $8.5970 \mathrm{e}-04$ & $4.7249 \mathrm{e}-04$ & $2.5799 \mathrm{e}-04$ & $1.3494 \mathrm{e}-04$ \\
\hline \multicolumn{6}{|c|}{ Midpoint Upwind scheme } \\
\hline $10^{0}$ & $9.4867 \mathrm{e}-04$ & $4.8465 \mathrm{e}-04$ & $2.4500 \mathrm{e}-04$ & $1.2318 \mathrm{e}-04$ & $6.1761 \mathrm{e}-05$ \\
\hline $10^{-1}$ & $3.7393 \mathrm{e}-03$ & $2.3894 \mathrm{e}-03$ & $1.4822 \mathrm{e}-03$ & $8.9549 \mathrm{e}-04$ & $5.2357 \mathrm{e}-04$ \\
\hline $10^{-2}$ & $3.4630 \mathrm{e}-03$ & $2.2148 \mathrm{e}-03$ & $1.3799 \mathrm{e}-03$ & $8.3307 \mathrm{e}-04$ & $4.8796 \mathrm{e}-04$ \\
\hline $10^{-4}$ & $3.4595 \mathrm{e}-03$ & $2.2122 \mathrm{e}-03$ & $1.3782 \mathrm{e}-03$ & $8.3203 e-04$ & $4.8735 \mathrm{e}-04$ \\
\hline $10^{-6}$ & $3.4595 \mathrm{e}-03$ & $2.2122 \mathrm{e}-03$ & $1.3782 \mathrm{e}-03$ & $8.3202 \mathrm{e}-04$ & $4.8734 \mathrm{e}-04$ \\
\hline $10^{-8}$ & $3.4595 \mathrm{e}-03$ & $2.2122 \mathrm{e}-03$ & $1.3782 \mathrm{e}-03$ & $8.3202 \mathrm{e}-04$ & $4.8734 \mathrm{e}-04$ \\
\hline \multicolumn{6}{|c|}{ Cubic Spline scheme } \\
\hline $10^{0}$ & $1.6943 \mathrm{e}-04$ & $9.3315 \mathrm{e}-05$ & $4.9098 \mathrm{e}-05$ & $2.5207 \mathrm{e}-05$ & $1.2774 \mathrm{e}-05$ \\
\hline $10^{-1}$ & $1.6098 \mathrm{e}-03$ & $7.4125 \mathrm{e}-04$ & $3.1441 \mathrm{e}-04$ & $1.6221 \mathrm{e}-04$ & $8.2062 \mathrm{e}-05$ \\
\hline $10^{-2}$ & $6.0150 \mathrm{e}-03$ & $3.4445 \mathrm{e}-03$ & $1.2304 \mathrm{e}-03$ & $4.3554 \mathrm{e}-04$ & $2.0100 \mathrm{e}-04$ \\
\hline $10^{-4}$ & $6.2040 \mathrm{e}-03$ & $3.9637 \mathrm{e}-03$ & $2.2137 \mathrm{e}-03$ & $1.1663 \mathrm{e}-03$ & $5.9776 \mathrm{e}-04$ \\
\hline $10^{-6}$ & $6.2039 \mathrm{e}-03$ & $3.9637 \mathrm{e}-03$ & $2.2137 \mathrm{e}-03$ & $1.1665 \mathrm{e}-03$ & $5.9831 \mathrm{e}-04$ \\
\hline $10^{-8}$ & $6.2039 \mathrm{e}-03$ & $3.9637 \mathrm{e}-03$ & $2.2137 \mathrm{e}-03$ & $1.1665 \mathrm{e}-03$ & $5.9831 \mathrm{e}-04$ \\
\hline
\end{tabular}


Table 4: $E^{N, M}$ and $r^{N, M}$ for Example (14) with $T=1.0, \delta=0.5 \times \varepsilon, N=M$

\begin{tabular}{lccccc}
\hline$\varepsilon \downarrow$ & $\mathrm{N}=32$ & $\mathrm{~N}=64$ & $\mathrm{~N}=128$ & $\mathrm{~N}=256$ & $\mathrm{~N}=512$ \\
\hline \multicolumn{7}{l}{ Proposed Hybrid scheme } \\
$E^{N, M}$ & $1.4572 \mathrm{e}-03$ & $8.6051 \mathrm{e}-04$ & $4.7258 \mathrm{e}-04$ & $2.5804 \mathrm{e}-04$ & $1.3496 \mathrm{e}-04$ \\
$r^{N, M}$ & 0.7599 & 0.8646 & 0.8730 & 0.9351 & 0.95380 \\
\hline \multicolumn{7}{l}{ Midpoint Upwind scheme } \\
$E^{N, M}$ & $3.7393 \mathrm{e}-03$ & $2.3894 \mathrm{e}-03$ & $1.4822 \mathrm{e}-03$ & $8.9549 \mathrm{e}-04$ & $5.2357 \mathrm{e}-04$ \\
$r^{N, M}$ & 0.64612 & 0.68891 & 0.72699 & 0.77429 & 0.83724 \\
\hline Cubic Spline scheme & & & & \\
$E^{N, M}$ & $6.2040 \mathrm{e}-03$ & $3.9637 \mathrm{e}-03$ & $2.2137 \mathrm{e}-03$ & $1.1665 \mathrm{e}-03$ & $5.9831 \mathrm{e}-04$ \\
$r^{N, M}$ & 0.6464 & 0.8404 & 0.9243 & 0.9632 & 0.98295 \\
\hline
\end{tabular}




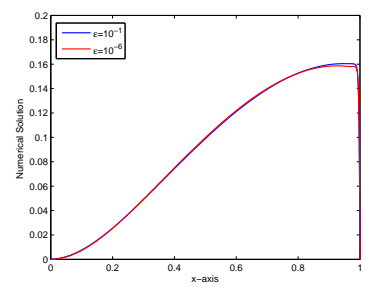

(a) Example 13

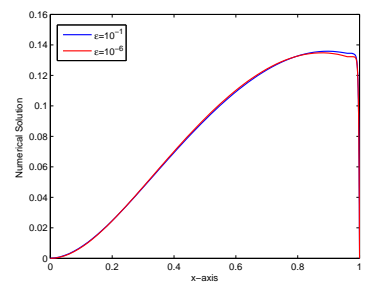

(b) Example 14
Figure 1: The behavior of solution for different values of of $\varepsilon$ at $T=1, \delta=0.5 \times \varepsilon, N=M=128$ for Examples 13 and 14.

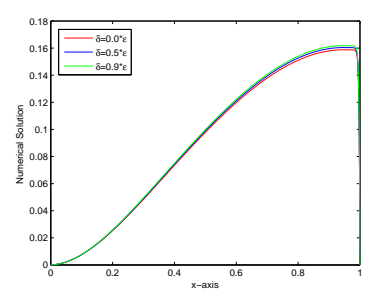

(a) Example 13

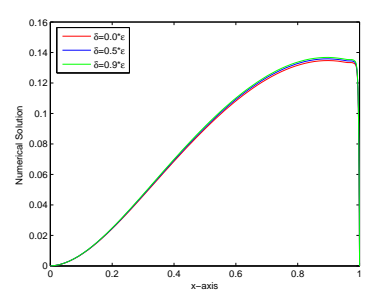

(b) Example 14
Figure 2: Effect of delay on the solution behavior for Examples (13) and 14 at $T=1, \varepsilon=10^{-1}, \eta=0.5 \times$ $\varepsilon, N=M=128$.

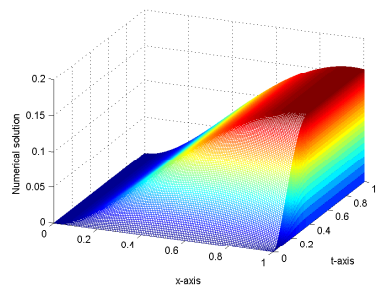

(a) Example13

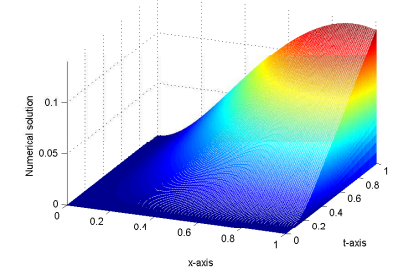

(b) Example 14
Figure 3: Numerical solution profiles for Examples (13) and 14 at $T=1.0, \delta=0.5 \times \varepsilon, \eta=0.5 \times \varepsilon$, $\varepsilon=10^{-6}, N=M=256$

The computed $E_{\varepsilon, \delta}^{N, M}, r_{\varepsilon, \delta}^{N, M}, E^{N, M}$ and $r^{N, M}$ for the test Examples 13, and 14 with the various values of $N, M$ and $\varepsilon$ with $\delta=\eta=0.5 \times \varepsilon$ are presented in Tables 1 - 4. From these tables, we can easily observe that the maximum pointwise errors decreases as the step sizes decrease for all values of the singular perturbation parameter, which confirm an $\varepsilon$-inform convergence of the proposed scheme. The proposed hybrid scheme provides more accurate numerical results than midpont upwind, cubic spline, and results in [9].

In Figures 1(a) and (b), the effect of the singular perturbation on the boundary layer behavior of the

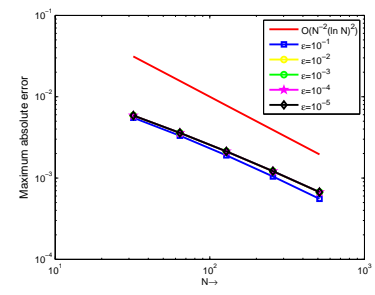

(a) Example13

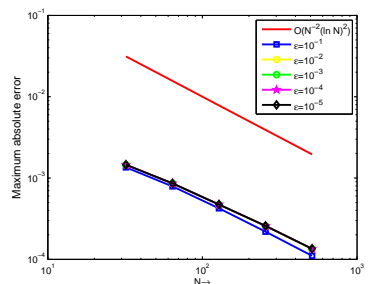

(b) Example 14
Figure 4: Log-log plot for Examples 13, and 14 respectively.

solution of Examples (13) and (14) respectively are shown for $T=1, \delta=0.5 \times \varepsilon$ and $N=M=128$. As observed in Figures (1) (a) and (b), when $\varepsilon \rightarrow 0$ strong boundary layer is formed near $x=1$. The influence of the delay parameter on the boundary layer behavior of the solution of Examples (13) and (14) is shown in Figures 2(a) and (b) respectively for $T=1, \varepsilon=10^{-1}$ and $N=M=128$. From Figures (2) (a) and (b), we observe that as the size of the delay parameter increases the thickness of the layer increases.

The 3D view of the numerical solution for Examples (13) and (14) are plotted in Figures (3) (a) and (b) respectively by taking $\varepsilon=10^{-6}, \delta=0.5 \times \varepsilon, N=$ $M=256$, which indicate the existence of the boundary layer near $x=1$. To depict the relationship between the $E_{\varepsilon, \delta}^{N, M}$ and the rate of convergence, we have used the log-log plot in Figures (4) (a) and (b) for Examples (13) and (14) respectively.

\section{CONCLUSION}

An $\varepsilon$-uniform numerical scheme is proposed for solving the singularly perturbed delay parabolic convection-diffusion equation. This scheme is developed based on the implicit Euler method for the temporal discretization with uniform step size and hybrid numerical scheme based on the midpoint upwind method in the coarse mesh regions and cubic spline difference method in the fine mesh regions on a piecewise uniform Shishkin mesh for the spatial discretization. The scheme is shown to be an $\varepsilon$-uniformly convergent of first-order in time and almost second-order in space directions. The efficiency of the scheme is shown by taking some test examples and comparing them with the numerical results obtained by midpoint upwind, cubic spline method, and results in [9]. It has been found that the proposed numerical scheme provides more accurate numerical results than the aforementioned methods. 


\section{REFERENCES}

[1] R. B. Stein, Some models of neuronal variability, Biophysical journal, 7,1967, pp.37-68.

[2] M. Derstine, H. Gibbs, F. Hopf, D. Kaplan, Bifurcation gap in a hybrid optically bistable system, Physical Review A, 26,1982,pp.3720.

[3] M. Wazewska-Czyzewska, A. Lasota. Mathematical models of the red cell system, Matematyta Stosowana, 6,1976,pp. 976.

[4] V. Glizer. Asymptotic solution of a boundaryvalue problem for linear singularly-perturbed functional differential equations arising in optimal control theory, Journal of optimization theory and applications, 106,2000, pp.309-335.

[5] A. Longtin and J. G. Milton. Complex oscillations in the human pupil light reflex with mixed and delayed feedback, Mathematical Biosciences, 90,1988,pp.183-199.

[6] V. Ramesh, M. K. Kadalbajoo. Upwind and midpoint upwind difference methods for timedependent differential difference equations with layer behavior, Applied mathematics and computation, 202,2008, pp.453-471.

[7] D. Kumar, M. K. Kadalbajoo. A parameteruniform numerical method for time-dependent singularly perturbed differential-difference equations, Applied Mathematical Modelling, 35,2011, pp.2805-2819.

[8] K. Bansal, P. Rai, K. K. Sharma. Numerical treatment for the class of time dependent singularly perturbed parabolic problems with general shift arguments, Differential Equations and Dynamical Systems, 25, 2017,pp. 327-346.

[9] D. Kumar. An implicit scheme for singularly perturbed parabolic problem with retarded terms arising in computational neuroscience, Numerical Methods for Partial Differential Equations, 34, 2018, pp.1933-1952.

[10] R. N. Rao and P. P. Chakravarthy. Fitted numerical methods for singularly perturbed onedimensional parabolic partial differential equations with small shifts arising in the modelling of neuronal variability, Differential Equations and Dynamical Systems, 27,2019, pp. 1-18.

[11] M. M. Woldaregay, G. F. Duressa. Parameter uniform numerical method for singularly perturbed parabolic differential difference equations, Journal of the Nigerian Mathematical Society, 38,2019, pp.223-245.

[12] I. T. Daba, G. F. Duressa. Extended cubic bspline collocation method for singularly perturbed parabolic differential-difference equation arising in computational neuroscience, International Journal for Numerical Methods in Biomedical Engineering, 37,2020.

[13] V. Ramesh, B. Priyanga. Higher order uniformly convergent numerical algorithm for timedependent singularly perturbed differentialdifference equations, Differential Equations and Dynamical Systems, 2019,pp.1-25.

[14] H. Tian. The exponential asymptotic stability of singularly perturbed delay differential equations with a bounded lag, Journal of Mathematical Analysis and Applications, 270,2002,pp. 143149.

[15] O. A. Ladyzhenskaia, V. A. Solonnikov, N. $\mathrm{N}$. Ural'tseva. Linear and quasi-linear equations of parabolic type, American Mathematical Soc., 23, 1968.

[16] G. I. Shishkin. A difference scheme for a singularly perturbed equation of parabolic type with discontinuous boundary conditions, USSR Computational Mathematics and Mathematical Physics, 28,1988, pp. 32-41.

[17] P. Mushahary, S. Sahu, and J. Mohapatra. A parameter uniform numerical scheme for singularly perturbed differential-difference equations with mixed shifts, Journal of Applied and Computational Mechanics, 6,2020),pp. 344-356.

[18] R. M. Priyadharshini, N. Ramanujam, and T. Valanarasu. Hybrid difference schemes for singularly perturbed problem of mixed type with discontinuous source term, J. Appl. Math. Info, 28, 2010,pp. 1035-1054.

[19] E. P. Doolan, J. J. Miller, W. H. Schilders, Uniform numerical methods for problems with initial and boundary layers, Boole Press, 1980.

\section{Creative Commons Attribution License 4.0 (Attribution 4.0 International, CC BY 4.0)}

This article is published under the terms of the Creative Commons Attribution License 4.0

https://creativecommons.org/licenses/by/4.0/deed.en_US 